\title{
Oxford circuits and systems conference
}

\author{
Bhaskar Choubey ${ }^{1,2} \cdot$ Khaled Hayatleh $^{3} \cdot$ Alex Pappachen James $^{4}$
}

Published online: 25 April 2018

(C) Springer Science+Business Media, LLC, part of Springer Nature 2018

The Oxford Circuits and Systems Conference (OXCAS) was organised on 19th of September 2017 as a reincarnation of the Analog Signal Processing Conference, which has been running biennially, in Oxford, since 1992. It began as a joint venture between Oxford Brookes University, Imperial College London and IBM Research UK. It has attracted a range of papers and speakers in analogue design from groups and individuals across the world. Past sponsors have included Analog Devices, Dia$\log$ Semiconductors, IBM Research UK, The IET, The IEEE, Saul Research, Texas Instruments UK and Toumaz Technology. The 2017 edition was organised jointly by the University of Oxford and Oxford Brookes University in the leafy campus of Somerville College, Oxford. Sponsored by PFU Imaging (Fujitsu) and the IET, the conference provided a free platform to engineers, students and researchers to exchange information and ideas. The conference does not charge a registration fee to ensure that attendees are not financially burdened. In addition, being a 1-day meeting, we hope to keep travel costs low as well.

The conference started with an invited speech by Prof. W. Rhett Davis of North Carolina State University, Rayleigh, USA on Physical Design of a Stacked Heterogeneous Multi-Core Processor. He showcased 3-d stacked processor design on Global Foundries $130 \mathrm{~nm}$ process and stacked

\footnotetext{
Khaled Hayatleh

khayatleh@brookes.ac.uk

Bhaskar Choubey

Bhaskar.choubey@eng.ox.ac.uk

Alex Pappachen James

alex.james@nu.edu.kz

1 University of Oxford, Oxford OX2 6HD, UK

2 University of Siegen, Siegen, Germany

3 School of Engineering, Computing and Mathematics, Faculty of Technology, Design and Environment, Oxford Brookes University, Wheatley Campus, Wheatley, Oxford OX33 1HX, UK

4 Nazarbayev University, Astana, Kabanbay Batyr, Astana 0001, Kazakhstan
}

using the Ziptronix face-to-face (F2F). 25 papers were then presented during the rest of the day, which covered almost the entire breadth of circuit design. At one end, we had papers on classical circuits like ADCs, Image Sensors, oscillators and amplifiers. At the other end, we had futuristic circuits with memristors and enabling Hierarchical Temporal Memory. New results in neural circuits, noise, mems devices and circuit layouts were also presented. Of these 12 of the papers have been selected after a double review process for selection in this special issue.

Oxford region has a long history of activity in electrical circuits and the conference reflects this longstanding tradition. Way back in 1929, a readership was established in the engineering laboratory of the University of Oxford. This attracted Eric Bailleul Moullin, later the president of Institute of Electrical Engineers. Moullin spent the next 10 years in Oxford reporting a number of studies on high frequency circuits, including temperature effects of inductors and condensors. The start of second world war led to Moulin working with Admiralty Signals Establishment and later Metropolitan-Vickers.

After the war, Oxford was also host to Rudolf Komfner's research on travelling wave tubes as microwave amplifiers. Amongst its other academics and alumnus, the university counts Robert Van de Graaf (High voltage generator bearing his name), Denys Wilkinson (Analogue to Digital Converter, bearing his name), Ralph Hartley (Oscillator bearing his name), Tim Berners Lee (Word wide web) and Tony Hoare (Quick Sort algorithm). In parallel to the University of Oxford, a second technical school was established in Oxford in 1891. Renamed as Oxford College of Technology in 1956, as Oxford Polytechnic in 1970 and finally as Oxford Brookes University in 1992, it had a significant contribution to the development of electrical engineering teaching in Oxford area. Francis John Lidgey, Emeritus professor there was instrumental in organising this conference in its early years as well as vital contributions to filter circuits. The current generation of academics at the two institutions hope to continue these traditions in future. 


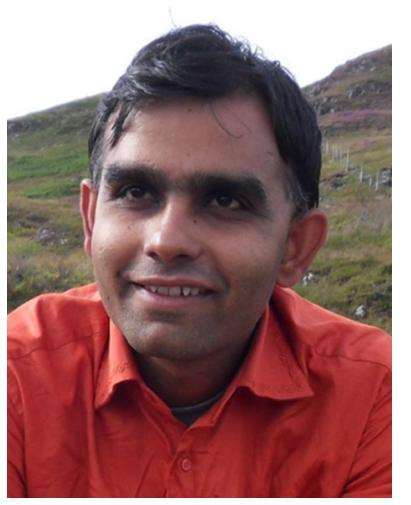

Bhaskar Choubey received the D.Phil. degree from the University of Oxford as a Rhodes Scholar, and the B.Tech. degree from the Regional Engineering College, Warangal, India. He is currently an Associate Professor of Engineering Science and a Fellow with Somerville College, University of Oxford, U.K., as well as chair-designate in Analogue Circuits and Image Sensors at the University of Siegen, Germany. He has been at the University of Glasgow, the Max Planck Institute of Brain Research, the University of Sydney, and North West University, South Africa. His research interests include CMOS image sensor, nonlinear dynamics, human visual system, and microelectromechanical systems. He received the IEEE Sensors Council GOLD Early Career Achievement Award, and the Myril B. Reed Best Paper Award from the IEEE Midwest Symposium of Circuits and Systems. He has served as an Associate Editor of the IEEE TRANSACTIONS ON CIRCUITS AND SYSTEMS II and the IEEE SENSORS JOURNAL.

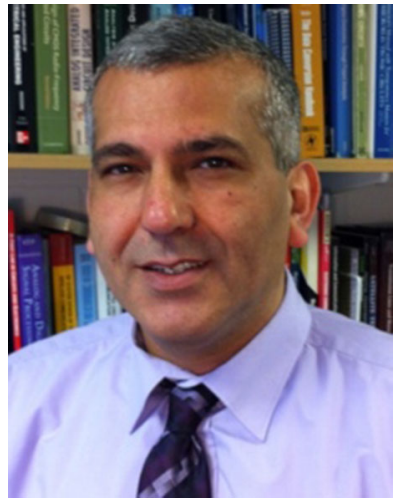

Khaled Hayatleh received his B.Eng. in June 1992 from Oxford Brookes University and subsequent Ph.D. in March 1996 from Oxford Brookes University in collaboration with Imperial College, London. His research interests are in the area of electronic circuits and systems for RF and biomedical applications. Current projects are in transconductance amplifier design for high frequency $\mathrm{RF}$ applications, amplifier linearisation, current feedback opamp development, automotive electronics, high accuracy voltage buffer and high output impedance current source design and a lowvoltage high-speed CMOS line driver for SerDes applications. He has over 100 publications in the above areas of research. He has been a keynote speaker at conferences in this area. Professor Hayatleh is currently the lead for electronics in the Biomedical Imaging and Instrumentation Research Team at Oxford Brookes University, UK.

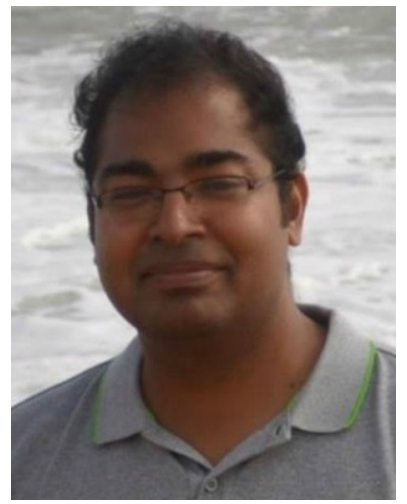

Alex Pappachen James received the Ph.D. degree from the Griffith University, Brisbane, QLD, Australia, which was completed in a short 2 years period in the area of hardware friendly bioinspired face recognition system. He works on the topics of memristive networks, neuromorphic computing and image processing for near-sensor edge devices. Currently, he is chairing the Electrical and Computer Engineering Department at Nazarbayev University. $\mathrm{He}$ is a mentor to several tech startups and co-founded companies in machine learning and computer vision hardware. He has been a founding chair for IEEE R10 Circuits and Systems Society Kerala Chapter, Executive Board Member of IET Vision and Imaging Network, founding chair of IEEE Kazakhstan subsection, R8 and mentor to IEEE NU Student Branch. He was an editorial member of Information Fusion (2010-2015), Elsevier, and is an Associate Editor for HCIS (2015-), Springer, IEEE Access (2017-), IEEE Transactions on Emerging Topics in Computational Intelligence (2017-), and IEEE Transactions on Circuits and Systems 1 (2018-). He was a faculty senate chair of NU between 2016 and 2017. He received an award from the president of Kazakhstan for his services to education in Kazakhstan in 2017. He is a Senior Member of IEEE, Life Member of ACM, and Senior Fellow of HEA. 